\title{
V2V Wireless Communication Protocol for Rear-End Collision Avoidance on Highways
}

\author{
Fei Ye, Matthew Adams, Sumit Roy \\ \{fye, mar22, sroy\}@u.washington.edu \\ Department of Electrical Engineering, University of Washington, Seattle, WA
}

\begin{abstract}
More than $23 \%$ of annual vehicle accidents are rear-end collisions, which provides an important test-case for enhanced collision avoidance approaches based on $\mathrm{v} 2 \mathrm{v}$ wireless communications. In this work, we propose and study the impact of a 802.11 based multi-hop MAC protocol that propagates an emergency warning message (EWM) down a platoon of cars on a highway. The design objective is to ensure reception of this message with stringent (low) delay constraints so as to provide drivers with requisite available maneuver time (AMT) to avoid rear-end collision. We provide realistic simulation studies of protocol performance within $\mathrm{ns}-2$ environment for various topology (1 lane and 3 lanes) and background traffic scenarios, as well as different protocol parameter settings, to highlight the potential of this approach for effective collision avoidance or mitigation.
\end{abstract}

\section{INTRODUCTION}

Since 1994, the number of fatal vehicle crashes in the United States annually has never fallen below 35,000 , and the number of persons killed in traffic accidents each year has consistently exceeded 39,000 [1]. Moreover, 23\% of all vehicle crashes (both fatal and nonfatal), or more than 1.5 million crashes per year, are rear-end collisions [2]. In Washington State, for the year 2005, rear-end crashes were the leading type of collision on state highways $(32.2 \%)$ compared to other major collision types such as fixed object (20.2\%) and sideswipes (8\%) [3].

The occurrence of a rear-end collision on a highway is a combined result of a lead vehicle's deceleration and a following vehicle's insufficient maneuvering time. Two major causes exist for a following vehicle's insufficient maneuvering time. First, drivers tend to keep shorter time-headway than is recommended in driver's manuals. Second, drivers often have a limited line-of-sight, which makes it difficult to anticipate hazardous conditions beyond the vehicle immediately in front. Therefore, when the driver's Needed Maneuvering Time (NMT) (driver reaction time plus the vehicle's response time) is greater than the Available Maneuvering Time (AMT), a rearend collision is inevitable. Studies [4] show that the driver's reaction time has a mean of $1.5 \mathrm{~s}$ and an $85^{\text {th }}$ percentile of $1.9 \mathrm{~s}$ , which dominates the NMT. Other studies [5] suggests that $60 \%$ of the rear-end crashes could potentially be avoided if the driver had an extra $0.5 \mathrm{~s}$. In most cases, to avoid a rear-end collision, the driver does not need to know detailed information about the situation beyond the lead vehicle. Simply warning of the potential hazard ahead would be enough.

Existing vehicle safety systems are based on various types of sensors (radar, vision sensors), which have a field-of- vision limited to immediate neighbors around the vehicle of interest. Therefore, these systems are not effective in providing drivers vision beyond the lead vehicle to avoid rear-end collision. Recently, the allocation of $75 \mathrm{MHz}$ in the $5.9 \mathrm{GHz}$ band for Dedicated Short Range Communication (DSRC) [6] has created ample opportunities for vehicle-to-vehicle (v2v) and vehicle to roadside (V2R) communication, where safety applications and rich media content delivery are enabled using low-cost commodity radios. In this paper, we study Emergency Warning Message (EWM) delivery in v2v multi-hop networks in order to prevent chain rear-end collisions on highways.

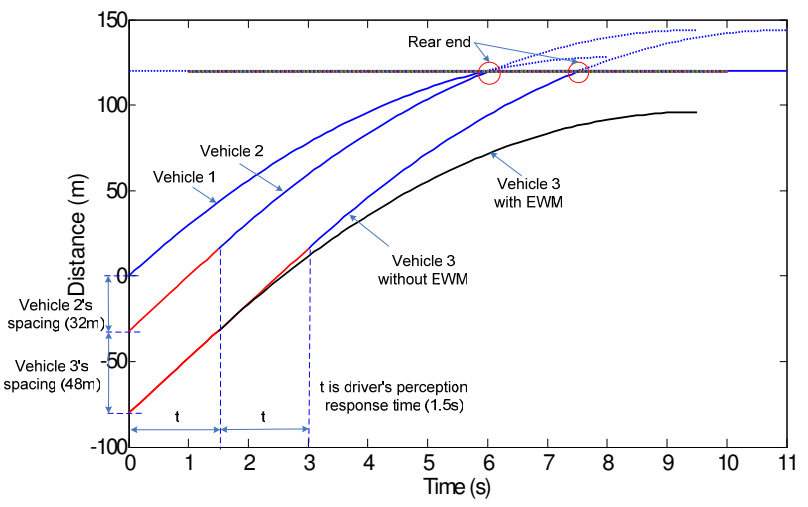

Fig. 1. Chain rear-end collision without EWM

Fig. 1 illustrates a chain rear-end crash involving 3 vehicles on a highway. In this example, all vehicles cruise at an identical speed of $32 \mathrm{~m} / \mathrm{s}(72 \mathrm{mph})$ and have the same deceleration of $4 \mathrm{~m} / \mathrm{s}^{2}$. The perception response time of all drivers are also identical (1.5s). Vehicle 2 follows vehicle 1 with an unsafe inter-vehicle spacing of $32 \mathrm{~m}$ (1s). Vehicle 3 keeps a safe spacing of $48 \mathrm{~m}(1.5 \mathrm{~s})$. Additionally, assume that vehicle 1 brakes at time 0 due to an emergency event. Without v2v communication, vehicle 2 and 3 start braking at $1.5 \mathrm{~s}$ and $3.0 \mathrm{~s}$ respectively. Vehicle 2 collides with vehicle 1 at the distance of $120 \mathrm{~m}$, and gets hit by vehicle 3 later. With a rear-end collision avoidance system, both vehicle 2 and 3 start braking at $1.5 \mathrm{~s}$, and vehicle 3 is saved. The observations here are:

- Without $\mathrm{v} 2 \mathrm{v}$ communication, the propagation of an emergency message along a platoon is delayed by each driver's perception response time.

- Being further away from vehicle 1 and keeping a relatively large inter-vehicle spacing with vehicle 2 do not 
guarantee vehicle 3's safety.

- A rear-end collision avoidance system greatly decreases the propagation delay of an EWM, therefore providing drivers more AMT to avoid collisions.

In this paper, we discuss the challenges in Medium Access Control (MAC) and network layer techniques, and identify the application requirements for a vehicular rear-end collision avoidance warning system. A novel rear-end collision avoidance $\mathrm{v} 2 \mathrm{v}$ wireless communication protocol is presented and evaluated by simulation.

\section{Challenges in Applying v2v Wireless Network TO COLlision AVOIDANCE}

Though similar, v2v wireless communication networks differ from the well-studied wireless ad hoc network in several ways, particularly related to Medium Access Control (MAC) and routing.

1) The anonymity problem. In a v2v wireless network, the addresses of vehicles on highways are unknown to each other. Although periodical broadcasts from each vehicle may inform direct neighbors about its address, the address-position map will inevitably change frequently due to lane changing, passing, leaving and entering the highway and other relative movements among vehicles. Additionally, in most safety applications, it is the receiver's responsibility to decide the relevance of emergency messages and decide on appropriate actions. Therefore, broadcast and multicast are the proper communication methods for collision avoidance. In fact, through integration with positioning systems, locationbased broadcast is the de facto method in most Cooperative Collision Avoidance (CCA) systems.

2) Multihop forwarding. Without any roadside infrastructure, multihop forwarding must be enabled to propagate the EWM along a platoon of vehicles. The ad hoc v2v network is different from traditional ad hoc networks in the following 2 aspects: first, no route setup is performed before forwarding; second, the EWM is sent as a broadcast rather than a unicast transmission.

3) Stringent delay requirement. A rear-end collision occurs when the AMT is less than the NMT. NMT is dominated by the driver's perception response time, which is determined by many factors, and therefore difficult to change. To effectively prevent a rear-end collision, a vehicle must receive the EWM a certain amount of time before the lead vehicle's deceleration to provide more AMT. The rear-end free condition is expressed as:

$$
t_{E W M}+T_{N M T}<t_{E W M}^{*}+T_{N M T}^{*}+T_{\text {headway }}
$$

where $t_{E W M}$ denotes the moment that the $i t h$ vehicle receives the EWM, $T_{N M T}$ and $T_{\text {headway }}$ are the $i t h$ vehicle's needed maneuver time and time-headway. * represents the lead vehicle $((i-1) t h)$. Assuming identical NMT, the EWM propagation delay from the $(i-1) t h$ to the ith vehicle must satisfies:

$$
\begin{aligned}
& T_{\text {delay }}=t_{E W M}-t_{E W M}^{*} \\
& T_{\text {delay }}<T_{\text {headway }}
\end{aligned}
$$

The intuitive explanations here are two folds. First, a more stringent delay constraint is required to save a careless driver who keeps a small inter-vehicle spacing. Second, the worst case is that the driver relies on the lead vehicle's braking light, which results in the maximum delay of $T_{N M T}^{*}$. Careful drivers who keep a time-headway over $T_{N M T}^{*}$ are always safe unless the lead vehicle hits a fixed object. Although transmitting an EWM packet corresponds to less than $1 \mathrm{~ms}$ in a clear wireless environment, dramatic delay increase is observed in crowded situations [8]. Due to multiple lanes, anti-parallel traffic, background ITS traffic, v2v networks should be considered dense wireless networks. Traditional wireless networking protocols should be enhanced to satisfy the delay constraint.

4) Redundant EWMs. Broadcast packets are not acknowledged. Therefore, periodic broadcasts are used to improve the probability of successful EWM delivery. However, two problems arise. First, a vehicle who has already successfully forwarded an EWM will keep contending with following vehicles for channel access. Second, redundant periodic broadcasts waste bandwidth and suppresses other data traffic. An implicit acknowledgement (ACK) strategy is adopted to eliminate redundant EWMs, in which the reception of an EWM from a subsequent vehicle in the platoon serves as an implicit ACK to vehicles in front. On receiving an implicit ACK, a vehicle immediately stops sending any EWMs related to the same event.

\section{RELATED WORK}

According to the challenges addressed above, existing protocol designs for collision avoidance in $\mathrm{v} 2 \mathrm{v}$ communication networks can be categorized into MAC protocol design and routing strategy design.

A set of slot reservation MAC protocols have been proposed for inter-vehicle communication [14]. Although, R-ALOHA makes the delay more predictable, it is an unsolved problem to achieve synchronization and slot allocation across multiple hops in the absence of a central controller. The IEEE 802.11a Distributed Coordination Function (DCF) based MAC protocol is preferred for its compatibility with commodity radio chips. However, several limitations in adopting DCF in v2v communication have been observed in [15]. Although the DCF is the de facto MAC layer in many simulation studies of $v 2 \mathrm{v}$ communication protocol design for safety applications [11], [13], [16], there is not sufficient in-depth understanding of how to satisfy the stringent delay constraint, especially in dense network scenarios.

Due to challenges 1, 2 and 4, the routing strategy in a v2v network should be broadcast-oriented and use location-based 
forwarding. Single hop broadcast strategies for safety application are discussed in [9]-[11]. [11] presents a congestion control algorithm to support multiple abnormal vehicles in the same contention area. A multihop broadcast protocol on the basis of slot reservation MAC is proposed in [12]. However, its design metric is the vehicle identification rate, which is a measure of connectivity in $\mathrm{v} 2 \mathrm{v}$ network. An intelligent multihop broadcast strategy with implicit acknowledgement is presented in [13] to achieve low EWM propagation delay.

Different from prior work, this paper focuses on an integrated protocol design and evaluation for rear-end collision avoidance on highways. Based on the discussion in section II, we propose a rear-end collision avoidance protocol which satisfies the stringent delay constraint. Both single lane and multi-lane scenarios are simulated.

\section{REAR-END COLLISION AVOIDANCE COMMUNICATION PROTOCOL}

In this section we present our rear-end collision avoidance protocol for a $\mathrm{v} 2 \mathrm{v}$ communication network. Each vehicle on the highway is assumed to be equipped with a positioning device (e.g. Global Positioning System) and an IEEE 802.11 radio working in ad hoc mode. Vehicles cruising in one lane have identical velocity and knowledge of their lane ID. There are multiple lanes, however, we assume no lane changing during the EWM propagation. When an emergency event occurs, the affected vehicle broadcasts an EWM to inform following peers. The warning message contains the sender's position, lane ID, event ID, event location, event time stamp, and message lifetime. Upon receiving such an EWM, the following vehicles inform their drivers of the potential hazard through an audio or visual signal. In such a way, drivers become aware of the emergency situations before they see the braking light of the lead vehicle. We further assume that all vehicles, upon receiving the EWM, start to decelerate after a pre-defined driver's perception response time.

\section{A. MAC Enhancement}

The MAC layer is based on standard IEEE802.11 DCF. To satisfy the stringent EWM propagation delay constraint, the following enhancement must be applied.

Whenever an EWM is generated, it is inserted to the head of the queue, but behind any former EWMs. Equivalently, a virtual queue is created for EWMs, which has absolute priority over regular queues. In DCF, to decrease collision probability, each transmission failure leads to doubled Contention Window (CW) size in the next backoff up to a maximum value. However, the probability of successful channel access rapidly decreases as the backoff stage increases. This may lead to unacceptable delay for some EWMs. To provide EWM higher probability of channel access, a fixed CW size is used for EWMs only. Whenever a channel access attempt fails for an EWM, the CW size is not doubled in the next backoff. Furthermore, an EWM has no retry limitation. The IEEE802.11e Enhanced Distributed Channel Access (EDCA)

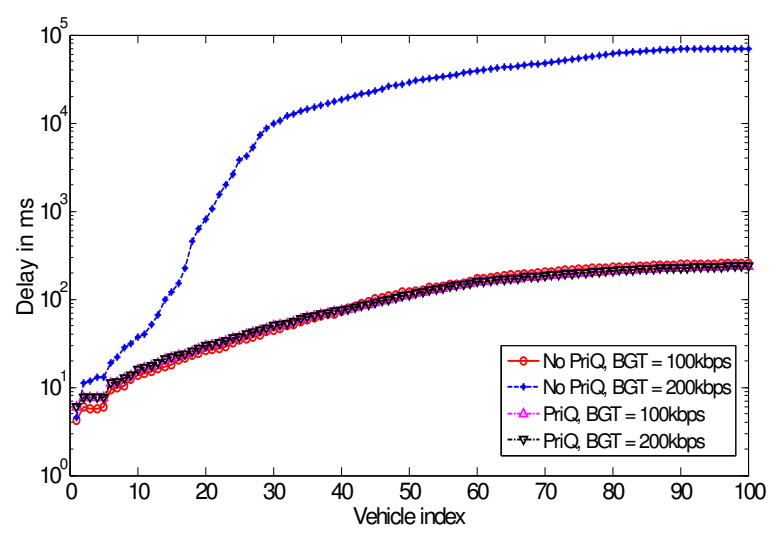

Fig. 2. The EWM propagation delay in single lane scenario with/without priority queue

[7] also provide QoS differentiation by different contention parameters (i.e. Arbitration Inter-Frame Space, different $\mathrm{CW}_{\text {min }}$ and $\mathrm{CW}_{\max }$ ), however, only in a statistical sense. So it is not appropriate for collision avoidance.

\section{B. Multihop Broadcast}

Due to the anonymity problem, EWMs are sent as broadcasts. Upon receiving an EWM, a vehicle accepts this warning message only if it comes from vehicles in front with the same lane ID, the event ID is new, and the message has not exceeded its lifetime. The vehicle immediately informs its driver and broadcasts a new EWM. Since group communication is not acknowledged in DCF, a sender should periodically broadcast until an implicit ACK is received. The implicit ACk is defined as an EWM with the same event ID from a subsequent vehicle in the same lane. This mechanism greatly reduces the redundancy. An EWM propagation stops when this message expires.

\section{Performance Evaluation of Rear-end collision AVOIDANCE}

The proposed rear-end collision avoidance protocol is implemented in the NS2 network simulator with proper modifications. The performance is evaluated in a single lane scenario and a 3-lane scenario with 100 vehicles in each lane. We assume low visibility on the freeway (i.e. rain, fog) such that each vehicle can only see one vehicle ahead. The first vehicle is forced to execute an emergency brake, which triggers an EWM message broadcast. In the 3-lane scenario, one EWM is generated and propagated in the central lane. For simplicity, vehicles are not allowed to change lanes. Basic parameters used in our simulation are summarized in Table I. In the following evaluation, several key parameters such as queue types, broadcast power, and background traffic are discussed.

\section{A. Performance in Single Lane Scenario}

In the single lane scenario, 100 vehicles are placed in a platoon with fixed time-headway (1s), which is less than the driver's perception response time (1.5s). Under the assumption that drivers can only see one vehicle ahead, all the vehicles will 


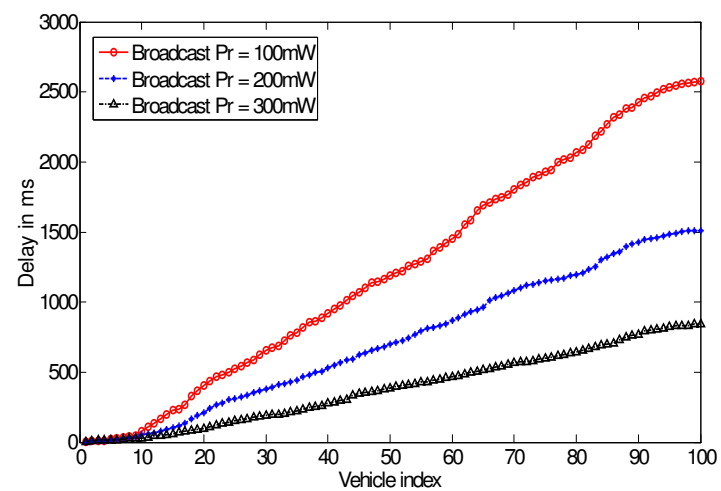

(a) EWM propagation delay under different broadcast power

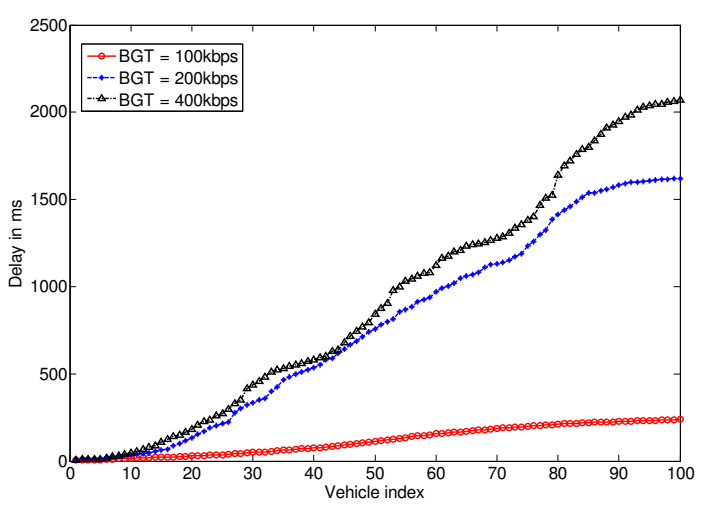

(b) EWM propagation delay under different background traffic

Fig. 3. The EWM propagation delay in single lane scenario under different broadcast power and background traffic (with priority queue).

eventually rear-end. By default, each vehicle has 400kbps unicast background data traffic, $200 \mathrm{~mW}$ EWM broadcast power, $200 \mathrm{~mW}$ unicast power, and a priority queue for EWMs. First, we compare the results with and without a priority queue in Fig. 2. Without priority queuing, the accumulated delay does not remarkably increase when background traffic is light (100kbps). But in the case of 200kbps background traffic, there is a large queuing delay for EWM, and the total delay increase dramatically to 70 seconds, which clearly does not meet the strict delay constraint for collision avoidance. Therefore, priority queueing is needed for vehicle safety applications.

According to Fig. 3(a) and Fig. 3(b), the EWM propagation delay in single lane scenario is extremely low regardless of the parameter settings as long as priority queuing is adopted. The accumulated EWM propagation delay for the whole platoon is always less than $2.5 \mathrm{~s}$, and the per vehicle latency is almost identical, and less than $30 \mathrm{~ms}$. Therefore, all vehicles are saved.

\section{B. Performance in 3-lane scenario}

In the 3-lane scenario, vehicles have a fixed reaction time $(1.5 \mathrm{~s})$ and uniformly distributed inter-vehicle spacing from $20 \mathrm{~m}$ to $45 \mathrm{~m}$. Without v $2 \mathrm{v}$ communication, statistically 70 rearend crashes will occur. By default, each vehicle has 100kbps unicast background data traffic, $200 \mathrm{~mW}$ EWM broadcast power, $100 \mathrm{~mW}$ unicast power, and priority queuing. The 3lane scenario is a much denser vehicular wireless network, thus larger accumulated delay than that in the single lane scenario is observed (Fig. 4). Both EWM broadcast power and the amount of background traffic impact the EWM propagation delay, however, the former dominates. Very few vehicles are endangered, even in the case of small EWM broadcast power (e.g. red line with circle mark in Fig. 4(a)). To understand this, we plot the per vehicle EWM propagation delay and timeheadway for every single simulation run. Fig.5 is a typical plot with $100 \mathrm{~mW}$ EWM broadcast power, $100 \mathrm{~mW}$ unicast power, and 100kbps background traffic per vehicle. According to our assumption, if the per vehicle EWM propagation delay exceeds its time-headway (i.e. the 38th vehicle in Fig.5), a rear-end collision occurs. It shows that the proposed rear-end collision avoidance protocol successfully saves more than $99 \%$ of vehicles in 3-lane scenario under the worst case assumption that each vehicle has a limited vision of one vehicle ahead.

\section{CONCLUSION}

In this paper, we have discussed the importance and challenges of using v2v wireless communication for vehicle safety applications. A stringent EWM delay constraint is identified as the key metric for protocol design. An integrated rear-end avoidance protocol is presented, which is based on 802.11 MAC and multihop broadcast. Simulation results from both single lane and multiple lane scenarios demonstrate that the EWM propagation delay in the proposed protocol satisfies the stringent delay requirements. With proper EWM broadcast power, more than $99 \%$ of vehicles are free of rear-end collisions, even in the dense multiple lane scenario plus the worst case visibility assumption.

TABLE I

SIMULATION PARAMETERS

\begin{tabular}{l|l} 
Parameter & Value \\
\hline Number of lanes & 1 or 3 \\
Platoon size & 100 vehicles \\
Vehicle Velocity & $25 \mathrm{~m} / \mathrm{s}$ \\
Time-headway & uniform distribution in $[0.8 \mathrm{~s}-1.8 \mathrm{~s}]$ \\
Inter-vehicle spacing & uniform distribution in $[20 \mathrm{~m}-45 \mathrm{~m}]$ \\
Deceleration & $5 \mathrm{~m} / \mathrm{s} / \mathrm{s}$ \\
Driver's perception response time & $1.5 \mathrm{~s}$ \\
Channel data rate & $2 \mathrm{Mbps}$ \\
EWM size & 128 Bytes \\
$\mathrm{CW}_{\text {min }}$ & 15 \\
CW & 1023 \\
Fixed CW for EWM & 15 \\
EWM lifetime & $30 \mathrm{~s}$ \\
EWM broadcast period & $50 \mathrm{~ms}$ \\
EWM process time & $0 \mathrm{~s}$
\end{tabular}

\section{REFERENCES}

[1] Fata Analysis Reporting System (FARS) http://www-fars.nhtsa.dot.gov Accessed on Nov. 10, 2007.

[2] The National Highway Traffic Safety Administration (NHTSA) http://www.nhtsa.dot.gov/ Accessed on Nov. 10, 2007.

[3] 2005 Annual State Highway Collision Data Summary. Washington State Department of Transportation (WSDOT). http://www.wsdot.wa.gov/mapsdata/tdo/accidentannual.htm Accessed on Oct. 10, 2007. 

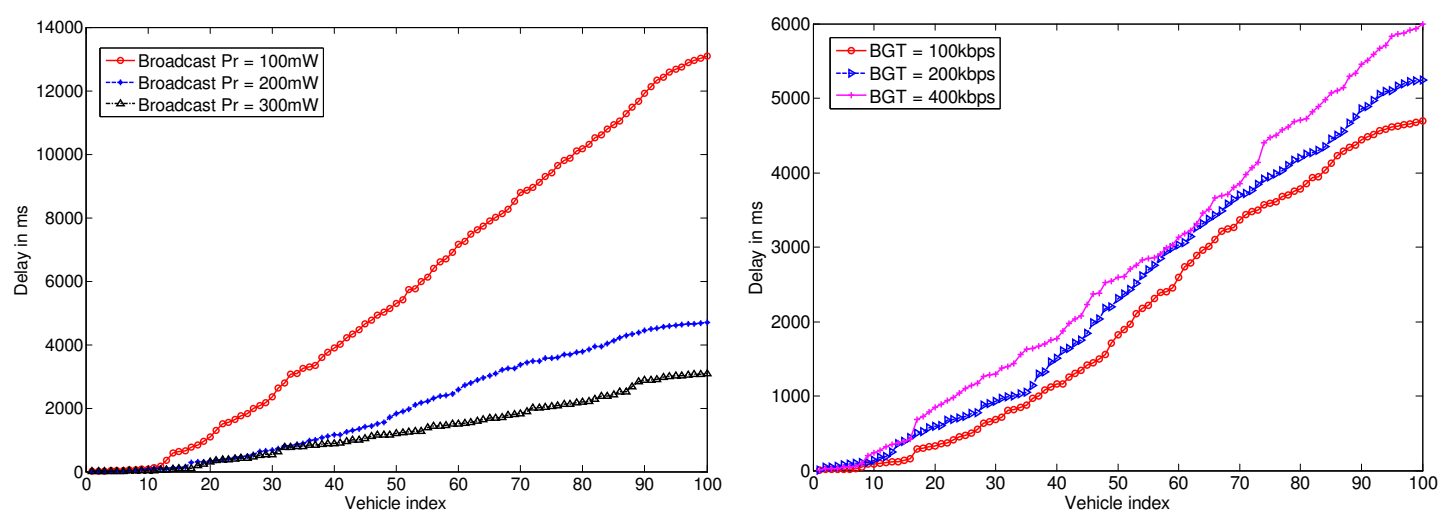

(a) The EWM propagation delay under different broadcast power (b) The EWM propagation delay under different background traffic

Fig. 4. The EWM propagation delay in 3-lane scenario (with priority queue).

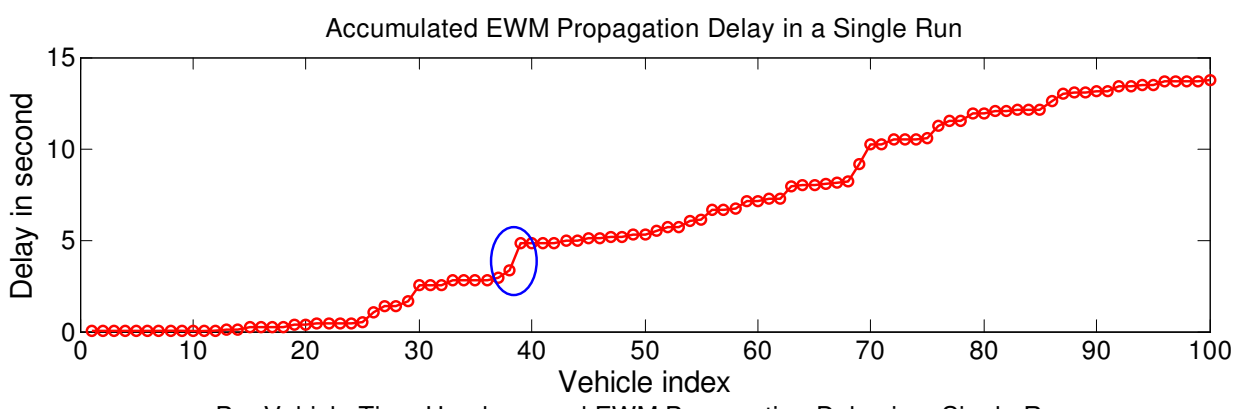

Per Vehicle Time-Headway and EWM Propagation Delay in a Single Run

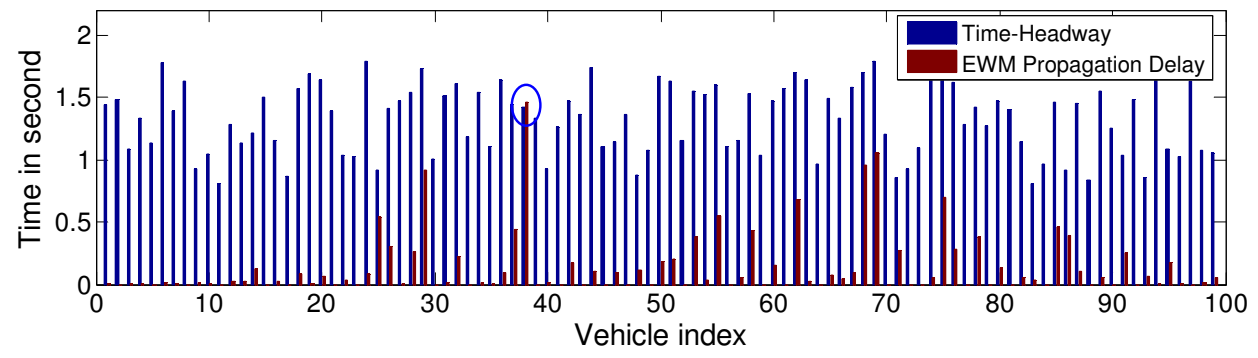

Fig. 5. Per vehicle EWM delay and time-headway.

[4] Lerner, Neil D., " Brake Perception-Reaction Times of Older and Younger Drivers," in Proc. of Human Factors and Ergonomics Society Annual Meeting Proceedings, pp. 206-210(5), 1993

[5] Final Report of Automotive Collision Avoidance Systems (ACAS) Program, The National Highway Traffic Safety Administration (NHTSA) www-nrd.nhtsa.dot.gov/pdf/nrd-12/acas/ACAS-FinalReport-2000-08.pdf Accessed on Aug., 2000.

[6] Standard Specification for Telecommunications and Information Exchange Between Roadside and Vehicle Systems-5GHz Band Dedicated Short Range Communications (DSRC) Medium Access Control (MAC) and Physical Layer (PHY) Specifications, ASTM E2213-03,2003

[7] IEEE 802.11e/D13.0, Part 11, Wireless LAN Medium Access Control (MAC) and Physical Layer (PHY) specifications: Medium Access Control (MAC) Enhancements for Quality of Service (QoS), 2005.

[8] Carvalho, M.M. and Garcia-Luna-Aceves, J.J., "Delay analysis of IEEE 802.11 in single-hop networks," in Proc. of 11th IEEE International Conference on Network Protocols (ICNP).

[9] Xu, Q., Sengupta, R. and Jiang, D., "Design and Analysis of Highway Safety Communication Protocol in $5.9 \mathrm{GHz}$ Dedicated Short Range Communication Spectrum," in Proc. of IEEE Vehicular Technology Conference-Spring, April 2003.

[10] ElBatt, T. and Goel, S.K. and Holland, G. and Krishnan, H. and Parikh, J., "Cooperative collision warning using dedicated short range wireless communications," in Proc. of the 3rd international workshop on Vehicular ad hoc networks, Sep., 2006, USA.

[11] Yang, X. and Liu, L. and Vaidya, NH and Zhao, F., "A Vehicle-toVehicle Communication Protocol for Cooperative Collision Warning," Technical Report, University of Illinois at Urbana-Champaign, Dec. 2003.

[12] Michael, L.B. and Nakagawa, M., "Non-Platoon Inter-Vehicle Communication Using Multiple Hops," IEICE Trans. Commun, Vol. 82, pp. 1651-1658, Oct., 1999.

[13] Biswas, S. and Tatchikou, R. and Dion, F., "Vehicle-to-vehicle wireless communication protocols for enhancing highway traffic safety," Communications Magazine, IEEE, Vol.44, No.1, pp.74-82, 2006.

[14] Verdone, R., "Multihop R-ALOHA for intervehicle communications at millimeterwaves," Vehicular Technology, IEEE Transactions on, Vol.46, No.4, pp.992-1005, 1997.

[15] Zhu, J. and Roy, S.,'MAC for dedicated short range communications in intelligent transport system," Communications Magazine, IEEE, 2003.

[16] Rabadi, N.M. and Mahmud, S.M., "Performance Evaluation of IEEE 802.11 a MAC Protocol for Vehicle Intersection Collision Avoidance System," Consumer Communications and Networking Conference, 2007. 